\title{
TRANSIZIONE DI REGIME E INTERESSI IMPRENDITORIALI IN ITALIA
}

\author{
di Massimo Ferrante
}

\section{Premessa}

Questo lavoro ha come obiettivo una prima analisi delle trasformazioni del sistema dell'associazionismo imprenditoriale a seguito dei numerosi e rilevanti effetti indotti dalla cosiddetta «transizione di regime» ${ }^{1}$ sui modelli di rappresentanza di interessi specifici e di regolazione sociale in Italia.

Utilizzando l'espressione sistema dell'associazionismo imprenditoriale intendo riferirmi alla pluralità di associazioni imprenditoriali oggi esistenti in Italia nei diversi settori produttivi, estendendo il campo dell'analisi al di là del caso della Confindustria, il referente empirico più noto e consolidato. Il mio sforzo maggiore sarà proprio orientato ad evidenziare le peculiarità della rappresentanza imprenditoriale che non si identifica con gli interessi della grande impresa tradizionale - piccola e media impresa, artigianato, commercio, agricoltura, cooperazione - limitando i richiami alla Confindustria (e ai sindacati confederali) per scopi comparativi. Credo di poter giustificare questa impostazione a partire da tre considerazioni preliminari.

La prima considerazione attiene alla letteratura oggi disponibile sull'organizzazione e sulle dinamiche proprie della rappresentanza degli interessi economici all'interno della più ampia sfera di analisi dei meccanismi di regolazione sociale. Nel caso italiano alla ricchezza della strumentazione teorica non corrisponde un'analoga completezza sul piano dell'indagine empirica. La ricerca è stata infatti pressoché monopolizzata dalle cicliche stagioni di studio sul sindacato e dalla sporadica attenzione

1 Per una disamina aggiornata della letteratura e delle analisi sulla transizione rimandiamo a Cotta e Isernia (1996).

RIVISTA ITALIANA DI SCIENZA POLITICA / a. XXVIII, n. 1, aprile 1998 
concessa alla Confindustria ${ }^{2}$ mentre ha sostanzialmente trascurato le altre associazioni imprenditoriali ${ }^{3}$.

Alcuni semplici dati di base, relativi al numero degli iscritti, evidenziano l'opportunità di colmare tale lacuna: se la Confindustria associa le più grandi imprese italiane (non solo private ma anche pubbliche), il $90 \%$ dei suoi iscritti è composto da piccole e medie imprese (su un totale di circa 100.000 imprese associate); la Confapi, che tutela la piccola industria, vanta circa 60.000 associati; nel settore dell'artigianato - che accoglie tra i suoi associati non solo artigiani, ma anche piccole e medie imprese -, la Confartigianato conta circa 400.000 iscritti e la Cna 350.000; nel settore del commercio, la Confcommercio registra più di 700.000 imprese iscritte e la Confesercenti poco più di 300.000; nel settore agricolo, la Coldiretti accoglie 650.000 imprese, quasi altrettante ne dichiara la Confagricoltura e circa 300.000 la Cia; nel settore della cooperazione, la Confcooperative conta oltre 20.000 imprese associate e la Lega delle Cooperative più di 10.000. Anche il solo dato della consistenza numerica suggerisce che si tratta di attori politici sicuramente rilevanti.

La seconda considerazione fa capo alla crescente valorizzazione sociale della piccola impresa. Il fenomeno non costituisce di per sé una novità; più recente ed originale è invece la legittimazione politica della piccola impresa. Essa ha conferito alla pluralità di istanze rappresentative delle varie forme di piccola impresa una rilevanza senza precedenti, che può essere colta, ad esempio, sulla base di alcuni elementi di larga evidenza. Possiamo ricordare, ad esempio, i cambiamenti registrati negli ultimi

2 Tra i pochi lavori centrati specificamente sull'organizzazione della rappresentanza imprenditoriale italiana si vedano: Urbani (1977), Addario e Segatti (1982), Lassini (1984), Windmuller e Gladstone (1985), Zan (1992), Martinelli (1994). Come approfondimento empirico o studio di caso sulla Confindustria: Speroni (1975), Lanzalaco (1990), Mattina (1991); sulla Lega delle Cooperative: Zan (1982); sul ruolo di Confindustria, Coldiretti e Confagricoltura nell'ambito del processo di consolidamento democratico i saggi di Mattina, Lanza e Morlino nel volume curato da Morlino (1991).

3 Mi sia concesso mettere in evidenza che l'Osservatorio sulle Associazioni di Rappresentanza dell'Università di Bologna - con cui chi scrive collabora attivamente - ha condotto negli ultimi anni una serie di ricerche che hanno avuto come referente empirico alcune delle associazioni imprenditoriali finora meno indagate nelle loro peculiarità; in tale contesto ho avuto inoltre occasione di prendere parte a numerosi momenti di confronto e dibattito con gruppi dirigenti nazionali e locali e, soprattutto, di essere osservatore partecipante in alcune fasi cruciali - ad esempio la campagna elettorale per le ultime elezioni politiche - ricavandone una serie di preziose informazioni dirette. Questo saggio si limiterà a trattare un aspetto specifico e circoscritto delle varie dimensioni analizzate in questi anni, ma è doveroso precisare da dove esso tragga origine. 
anni nelle relazioni tra governi e associazioni imprenditoriali «minori» (quelle cioè che rappresentano prevalentemente la piccola impresa). Durante il governo Ciampi e poi, soprattutto, con il governo Berlusconi, esse sono state presenti, sentite, convocate in misura quasi superiore all'intera fase repubblicana precedente, fenomeno che non può essere ricondotto unicamente alla natura tecnica dei governi Ciampi e Dini e alle difficoltà di «navigazione» del governo Berlusconi. Molte di queste associazioni, in secondo luogo, sono state in grado di imporre, nel corso della campagna elettorale per le elezioni politiche del 1996, larga parte dei temi in agenda, tanto è vero che gli schieramenti in campo hanno stilato programmi nei quali la specificità dei singoli settori di interesse emergeva con una puntualità mai verificata in passato: il Polo e l'Ulivo hanno entrambi destinato all'artigianato e alla piccola e media impresa una specifica sezione del loro programma; la Lega una sezione congiunta ${ }^{4}$. Il governo Prodi ha fin qui adottato la stessa linea dei tre governi precedenti, contribuendo a intensificare i canali di confrontos.

Dietro gli esempi appena menzionati stanno in realtà più sostanziali ragioni di carattere economico e politico. Le ragioni economiche, nelle quali non ci addentreremo, si possono evincere dall'enfasi e dall'attenzione con cui negli ultimi anni si è dibattuto della modernità e vivacità della piccola impresa come attore protagonista dello sviluppo economico nelle aree oggi più evolute del paese. Le ragioni di carattere politico sono invece legate ad un fenomeno che molti osservatori (Bordogna e Provasi 1984; Feltrin e Zan 1995; Salvadori 1996) considerano sempre più rilevante. Mi riferisco alla cosiddetta «economicizzazione della politica», al fatto, cioè, che le tematiche economiche (in primis l'Europa e il lavoro) costituiscono ormai sempre più le issues fondamentali del dibattito politico e definiscono aspettative e parametri di valutazione dell'operato del decisore pubblico. Le cause di tale evoluzione sono molteplici: dalla crisi delle forme più pure ed oltranziste dell'ideologia liberista (so-

4 Qualcuno ricorderà l'imbarazzante querelle, enfatizzata da tutti i mass media, sulla riproduzione testuale di alcuni passi di un più o meno formale documento della Confcommercio in un passaggio dei programmi elettorali dei due principali schieramenti.

5 Mi sia consentito citare, a mo' di aneddoto, lo stupore e le difficoltà organizzative recentemente confessatemi dai dirigenti nazionali di alcune associazioni imprenditoriali, non attrezzate come la Confindustria e i sindacati confederali ad un confronto continuo col governo, quando si sono trovati a dover fronteggiare reiterate convocazio$\mathrm{ni}$, anche nella stessa giornata e da parte di differenti ministeri. 
prattutto alla luce dei risultati conseguiti dai governi Thatcher e Reagan), alla globalizzazione dei mercati, alla conseguente necessità di ripensare il ruolo dello Stato nel governo dell'economia, alla ridefinizione degli assetti politico-economici europei. L'economicizzazione della politica favorisce, evidentemente, una valorizzazione del tessuto associativo che rappresenta gli interessi economici e attribuisce loro legittimazione e competenza. La rilevanza assunta dall'incentivazione della piccola imprenditorialità e del lavoro autonomo sul terreno delle politiche del lavoro e per l'occupazione - in quanto alternativa più plausibile al lavoro dipendente nella grande impresa o nel settore pubblico - costituisce, infine, un ulteriore aspetto di attualità e rilevanza della parte storicamente meno influente e meno visibile dell'associazionismo imprenditoriale.

Veniamo alla terza ed ultima considerazione preliminare. Abituata a considerare i soggetti della rappresentanza prevalentemente per il loro «peso specifico» all'interno dell'arena politi$\mathrm{ca}$, ossia per la loro rappresentatività, la scienza politica ha teso a trascurare la dimensione strutturale ed organizzativa degli interessi, $\mathrm{i}$ quali, in realtà, proprio a partire da tale dimensione prendono forma, confini e sostanza. Così procedendo si corre il rischio di incorrere nel «pregiudizio sociologico» evidenziato da Panebianco (1982) e di conferire agli interessi una «naturalità» che non possiedono. Questo richiamo ci è utile a due scopi: anzitutto per premettere che la «transizione di regime» degli anni ' 90 ha innescato un complesso, e tuttora incompleto, processo di ridefinizione di identità e confini all'interno del sistema della rappresentanza imprenditoriale, del quale i settori economici sopra ricordati, imperniati sulla centralità della piccola impresa, sono tra i principali protagonisti; in secondo luogo per sottolineare come la transizione trovi elementi forti di spiegazione non solo nelle strategie politiche, ma anche nei vincoli organizzativi storicamente acquisiti dalle associazioni: giova ricordare che molte associazioni imprenditoriali (a somiglianza dei sindacati confederali) sono organizzazioni estremamente complesse, tali non solo per il numero di iscritti ma anche, ad esempio, per il personale impiegato e le risorse economiche generate. Nel settore dell'artigianato - dove convivono quattro associazioni di rappresentanza (Cna, Confartigianato, Casa e Claai) - Cna e Confartigianato, le due associazioni più importanti, contano più di 12.000 dipendenti. Non è ancora possibile, allo stato attuale delle ricerche, formulare una stima delle entrate economiche 
complessive di ciascuna di esse. Disponiamo tuttavia di dati relativi a molte periferie, ossia ad associazioni provinciali di primo grado interne alle Confederazioni nazionali, da cui risulta, ad esempio, che la Cna di Bologna, con poco più di 500 dipendenti, ha entrate, tra tesseramento e servizi resi agli associati, per circa 60 miliardi all'anno e che la Confartigianato di Modena ha più di 400 dipendenti ed entrate superiori ai 35 miliardi all'anno. Non si tratta, dunque, di attori politici riconducibili ai pochi volti noti agli addetti ai lavori, ma di organizzazioni complesse, caratterizzate da una straordinaria multiformità delle ragioni di scambio con gli iscritti e con il contesto sociale, economico e politico. Le loro scelte nell'arena politica dipenderanno dunque anche da fattori di questo tipo.

Nei prossimi paragrafi prenderemo quindi in considerazione le associazioni imprenditoriali in quanto attori politico-istituzionali, mettendo in luce l'impatto che i recenti mutamenti del sistema politico hanno avuto sul sistema della rappresentanza imprenditoriale e sui meccanismi che hanno regolato i reciproci scambi nel recente passato. Analizzeremo le associazioni come attori organizzativi, focalizzando l'attenzione su alcune dimensioni cruciali per la comprensione delle dinamiche in atto, per poi presentare in sintesi le questioni aperte, che rendono difficile ipotizzare le possibili evoluzioni del sistema della rappresentanza funzionale in Italia.

Le associazioni imprenditoriali e l'arena politica: il modello del collateralismo

La caratteristica fondamentale dell'attuale assetto della rappresentanza imprenditoriale in Italia consiste nella sua elevata frammentazione strutturale. Le ragioni di tale frammentazione sono note e risiedono nella imposizione di confini organizzativi tra differenti associazioni a partire da alcuni assi che si sono storicamente sovrapposti e parzialmente intersecati. Di essi, quattro sono prevalenti, pur se non esaustivi: l'identità politica, il settore produttivo, la dimensione strutturale, la natura giuridica dell'impresa ${ }^{6}$. Fra questi, l'identità politica assume partico-

6 Prendiamo il caso, emblematico, di un piccolo imprenditore manifatturiero con una decina di dipendenti: a seconda delle aree geografiche, delle caratteristiche settoria- 
lare rilevanza, poiché si è configurata come spartiacque di singole categorie produttive omogenee. $\mathrm{E}$ in ragione di ciò che in Italia, in molti dei settori economici più rilevanti, esiste quantomeno una duplicazione delle rispettive organizzazioni di rappresentanza, storicamente dettata dal colore del partito politico di riferimento. Si tratta di un fenomeno che possiamo denominare collateralismo politico. Le sue implicazioni per il più generale funzionamento della democrazia e delle istituzioni sono state oggetto di un'ampia riflessione (Sartori 1966 e 1982; Amato 1976; Pasquino 1987; Pizzorno 1993); nel caso italiano, la sua rilevanza come meccanismo regolativo in sé è stata invece poco indagata ${ }^{7}$.

Di solito, si definisce collaterale un'associazione di rappresentanza, o comunque un gruppo di pressione, che è emanazione diretta di un partito politico e che ne costituisce quindi una sorta di articolazione organizzativa rispetto ad una determinata sfera di interessi. Nel caso italiano il concetto potrebbe sembrare non totalmente appropriato a descrivere $\mathrm{i}$ rapporti storicamente intercorsi tra Dc e Pci, da un lato, e le associazioni di rappresentanza, dall'altro, se non altro perché l'interpretazione più comunemente accettata del loro rapporto è quella centrata esclusivamente sullo scambio politico-elettorale. La questione può essere precisata a due livelli: il primo, più scontato, è quello del rapporto tra i singoli partiti e le singole associazioni; il secondo è quello aggregato del sistema complessivo di regolazione che si è storicamente creato, e che costituisce il dato davvero peculiare del caso italiano.

li, della cultura politica locale, dei servizi associativi di cui l'azienda necessita o della semplice propensione personale dell'imprenditore, egli potrà scegliere di iscriversi alla Confindustria, alla Confapi, alla Confartigianato o alla Cna, per citare le sole associazioni maggiori che oggi si offrono di rappresentarlo e/o di offrirgli servizi specifici. Un albergatore potrà essere iscritto alla Confindustria oppure alla Confcommercio, alla Confesercenti, alla Lega delle Cooperative, alla Confcooperative o financo alle varie associazioni dell'artigianato o, naturalmente, ad una piccola associazione di albergatori e pubblici esercenti di carattere locale. In realtà non vi è oggi in Italia un'associazione imprenditoriale che monopolizzi significativamente la rappresentanza di un settore: la Confindustria monopolizza sì la rappresentanza della grande impresa, ma la sua base sociale è in realtà composta prevalentemente di Pmi, terreno su cui è in aspra competizione con molte altre associazioni; significativa, al proposito, è stata la recente elezione a presidente degli industriali di un piccolo imprenditore come Giorgio Fossa.

7 Rispetto all'accezione comune, l'impiego del termine «collateralismo» potrebbe risultare fuorviante. Tuttavia, nell'indisponibilità di altri termini consolidati, ho preferito utilizzare egualmente il concetto, precisando al meglio in quale accezione e con che limiti. 
Sul piano del rapporto tra partito ed associazione ci troviamo di fronte ad un modello «puro» di collateralismo quando si verificano tre condizioni: $i$ ) il partito concorre a far nascere e a costituire l'associazione; ii) il partito controlla, mantenendoli nel tempo, i meccanismi di selezione e legittimazione dei gruppi dirigenti dell'associazione; iii) il partito determina le scelte fondamentali dell'associazione circa l'azione di rappresentanza degli interessi. Con differenze di grado, tutte e tre le condizioni si verificano nel caso italiano. In relazione alla nascita, se è vero che quasi tutte le principali confederazioni nazionali nei vari settori produttivi vengono fondate con le loro attuali denominazioni nell'immediato dopoguerra, è altrettanto vero che nascono con pochi iscritti e quasi senza articolazione territoriale: sono invece Dc e Pci a provvedere a ciò negli anni successivi, sebbene con due modelli tendenzialmente diversi. Il Pci inviava letteralmente «in missione» propri funzionari nelle varie aree del paese per aggregare e far nascere associazioni provinciali di agricoltori, artigiani, commercianti, cooperatori; la Dc, pur facendo ricorso anch'essa a tale modalità, tendeva soprattutto a cooptare $\mathrm{i}$ 《notabili» in grado di aggregare, in ambito locale, istanze rappresentative nei vari settori ${ }^{8}$. Tanto il modello del «commissario politico» che quello del «notabile» instauravano una ferrea logica di controllo sulla selezione della dirigenza associativa: la competizione all'interno delle associazioni era quella propria dei sistemi chiusi, nei quali i conflitti potevano essere solo quelli tra componenti e correnti interne ai partiti o tra partiti alleati.

In merito alla natura delle azioni e delle scelte di rappresentanza, conviene spostare il ragionamento sul piano sistemico. In questo caso, infatti, il nodo centrale non è la mera vicinanza politica di un'associazione o di un gruppo di interesse ad un partito - cosa che accade con differenti gradi di intensità in molte democrazie, dalla Germania agli Stati Uniti - quanto, piuttosto, che l'identificazione politica di specifici interessi economici abbia coinvolto la maggior parte della struttura produttiva del paese e si sia configurata in sé come meccanismo portante della regolazione degli interessi, dando luogo ad una variante davve-

8 Mi si perdonerà la ricostruzione, a dir poco frettolosa, di meccanismi che sarebbero un interessante oggetto di ricerca. Comunque in varie indagini sul campo, in associazioni di settori diversi e in diverse zone d'Italia, ho intervistato funzionari e dirigenti di lunga militanza che mi hanno confermato le due logiche esposte. 
ro particolare del modello della pressione pluralistica nella produzione delle politiche pubbliche.

Ciò è stato possibile in virtù di alcuni fattori specifici. Il primo di essi ha caratterizzato il sistema politico italiano fino a tempi recentissimi. Si tratta della sua configurazione di sistema polarizzato, ad alta conflittualità ideologica e «bloccato» nell'alternanza fra opposti schieramenti di governo. Il secondo fattore è il graduale e progressivo processo che trasforma il regime pluralista in un regime consociativo (Lijphart 1969; Pizzorno 1993), regime in cui l'opposizione rinuncia alla sua funzione istituzionale in cambio di un sostanziale coinvolgimento sul piano del processo decisionale e dell'allocazione delle risorse. In tale quadro è sempre stato il partito a prevalere sulle sedi istituzionali legislative ed esecutive come luogo privilegiato ove produrre mediazione degli interessi e regolazione sociale, ma lo spartiacque ideologico e la forte contrapposizione di valori non negoziabili (Pizzorno 1983) hanno fatto sì che $\mathrm{i}$ due maggiori partiti - Dc e Pci - abbiano potuto polarizzare attorno a sé l'intera galassia degli interessi funzionali di carattere non solo economico ma anche - non dimentichiamolo - culturale, sociale e ricreativo. Ne è derivata una duplicazione di rappresentanza delle principali categorie economiche - eccezion fatta per la grande impresa - e la contrapposizione tra piccoli imprenditori, artigiani, commercianti, agricoltori, cooperatori «bianchi» e «rossi».

Sostenere che il collateralismo italiano ha costituito una variante assai peculiare del modello di competizione pluralistica ${ }^{9}$ implica riconoscere la specificità che gli deriva dal combinare $\mathrm{i}$ tre modelli «puri» di regolazione degli scambi: il mercato, la gerarchia e il clan (Williamson 1975; Ouchi 1980). Ricordiamo brevemente le caratteristiche dei tre modelli: l'efficienza del mercato necessita della misurabilità delle prestazioni dei soggetti che scambiano e può ignorare la misura della congruenza sui fini particolari o della dose di opportunismo con cui essi accedono allo scambio; l'efficienza del clan, di converso, necessita di un'elevata congruenza sui fini dello scambio e può tollerare anche la bassa misurabilità delle reciproche prestazioni; la ge-

9 Pizzorno ha una posizione ancora più netta: «quando le risorse associative - costituite dalla capacità degli individui di impegnarsi in cause collettive (...) è totalmente, o quasi, convogliata attraverso canali politici, si ha di fatto, anche se l'apparenza può essere diversa, una paralisi del pluralismo» $(1993,291)$. 
rarchia, nella riformulazione di Williamson proposta da Ouchi, è un modello intermedio tra mercato e clan che demanda alla norma ed al principio di autorità la regolazione di scambi tra soggetti i cui fini non combaciano in maniera sufficiente, dando luogo a comportamenti opportunistici e a prestazioni non adeguatamente misurabili.

L'elevatissima capacità di persistenza del modello del collateralismo italiano si è per lunghi anni imperniata sul ruolo del partito, non solo in quanto soggetto regolatore delle scelte pubbliche, ma anche come fattore di integrazione e di «pedagogia» sociale. Con ciò, il partito ha mediato i conflitti strutturali imposti dalla pluralità di cleavages socio-economici esistenti e ha diretto, educato e controllato la società (tramite cellule, sezioni, parrocchie, circoli, movimenti e, ovviamente, associazioni) fin nella sfera del «privato» dei cittadini, in maniera tanto più pervasiva quanto meno incisivo è stato in essa il processo di «secolarizzazione» ideologica (Cartocci 1990 e 1994). Il rapporto tra partito ed associazioni collaterali, pertanto, non deve essere ricondotto sul mero piano dello scambio politico-elettorale pure innegabile - in un'ottica di mercato, oligopolista o pluralista a seconda delle fasi storiche o delle singole politiche pubbliche (Lange e Regini 1987). Del collateralismo è infatti importante evidenziare il carattere di meccanismo di creazione e mantenimento di coesione sociale - ossia di un «ordine» con valenza organizzativa (Friedberg 1993) - ovvero di una «armonia sistemica» (Feltrin e Zan 1995) che ha oltrepassato non solo la logica di scambio di mercato ma anche la stessa valenza simbolica o assiologica dell'identificazione collettiva per divenire meccanismo di normazione, emancipazione, giustificazione e garanzia degli scambi tra le svariate componenti della società. Un meccanismo, insomma, su cui si è fondata la coerenza della struttura sociale ed istituzionale italiana, in grado di mantenere a lungo l'equilibrio sistemico, anche se non in misura tale da difendersi da effetti perversi sul piano economico (la dilatazione della spesa pubblica) e dal processo entropico della secolarizzazione e dell'indebolimento delle grandi identità collettive.

All'interno di questa visione risulta più chiara la natura del rapporto tra partito ed associazioni collaterali: ove il primo ha sempre assunto la delega a rappresentare gli interessi verticali e le seconde, come corrispettivo per questa sua funzione di valorizzazione delle componenti categoriali della società, lo hanno legittimato come un potente meccanismo di «gerarchia delega- 
ta». La delega in questione ha avuto connotazioni originarie fortemente claniche che hanno assunto col tempo - sia in ragione della progressiva secolarizzazione, sia per comportamenti partitici sempre più opportunistici (sfociati alla fine in «Tangentopoli») - un profilo più propriamente burocratico-normativo o della pura «interdipendenza dei privilegi» tra i gruppi dirigenti (Crozier e Friedberg 1977). Se vogliamo, detto in altri termini, la legittimazione della gerarchia partitica ha via via esaurito le sue componenti valoriali in seno alla società e, perdendo la capacità di generare integrazione sociale, si è ritrovata circoscritta, nell'ultima fase che giunge alla crisi di regime, alla ristretta cerchia delle oligarchie organizzative ed alle loro strategie di sopravvivenza. Da questo punto di vista si possono meglio comprendere i rapporti tra partito e dirigenza associativa e il perché l'influenza del partito sulle associazioni abbia investito la creazione delle élites, la designazione degli incarichi, la fissazione dei criteri di controllo, valutazione e formazione, configurandosi quindi come una sorta di efficiente e potente «direzione del personale» nel senso più pieno e meno tecnicistico della funzione ${ }^{10}$. Si chiarisce infine perché all'interno di tale meccanismo la possibilità per le associazioni di optare per strategie di accentuazione della propria autonomia sia stata non soltanto non conveniente ma nemmeno ipotizzabile ${ }^{11}$.

Alla luce di quanto finora argomentato possiamo riassumerne le cause in quattro ragioni di fondo:

10 È importante precisare che il riferimento alla delega di rappresentanza concessa dai gruppi ai partiti o, di converso, alla gerarchia del partito sui gruppi non va inteso in senso meccanicistico, ad esempio confondendo tale concettualizzazione con la vecchia teorizzazione (politologicamente «rozza»: cfr. Morlino 1991) delle «cinghie di trasmissione», difficilmente adattabile per l'area Dc e, dalla fine degli anni '60, sempre meno applicabile anche per l'area Pci. La storia delle singole associazioni lo dimostra. Senza voler chiamare in causa il troppo facile esempio del sindacato, si può far riferimento alla cooperazione «rossa»: nel momento dell'apertura del Pci ai ceti medi non solo all'interno della cooperazione di consumo nasce il Conad, ma la stessa volontà del Pci era di trasformare tutte le cooperative di consumatori in cooperative di dettaglianti e «conadizzazione» era la parola d'ordine dell'epoca. La storia, grazie alla forte contrapposizione dei dirigenti cooperativi comunisti e socialisti, è andata in modo diverso (mi permetto di rinviare a Ferrante 1993). Ed altri esempi sarebbero possibili in altri settori. Pertanto parliamo di «gerarchia delegata» con ciò intendendo un modello pervasivo di integrazione sociale al cui interno era il partito, tramite le sue anime ed articolazioni, a farsi onere della mediazione tra interessi comunque contrapposti.

11 In questo senso sono d'accordo con Pasquino (1987) quando scrive che gruppi sufficientemente forti da articolare direttamente $\mathrm{i}$ propri interessi hanno in realtà sempre optato per eleggere loro rappresentanti parlamentari nel partito, come ad esempio Coldiretti o Confcommercio con la Dc. 
a) la polarizzazione ideologica: l'identità associativa era data da una concezione totalizzante del mondo e della società e l'espressione corporativa degli interessi era giustificata all'interno di tale concezione, con un minimo o nullo grado di indipendenza rispetto al partito;

b) i meccanismi di regolazione pubblica: non solo tutte le associazioni godevano in qualche misura della rendita di posizione concessa dal regime consociativo, ma presupposti e contenuti del policy making - come hanno argomentato efficacemente, tra gli altri, Cotta e Isernia (1996) - erano coerenti col più funzionale presidio di tale assetto ponendosi o al livello di «metapolitiche» (ad elevato tasso ideologico e simbolico) o al livello di «micropolitiche» (piccoli provvedimenti ad alto tasso distributivo) e minimizzando invece le «mesopolitiche» (le politiche di riforma di più ampia portata);

c) l'identificazione dei gruppi dirigenti associativi con le oligarchie partitiche;

d) la pervasività sociale e le logiche di funzionamento del modello collateralista che implicavano lo scardinamento dei più tradizionali meccanismi di scambio tra soggetti della lobbying e decisore pubblico. Sappiamo, infatti, che nella concezione più classica la possibilità per un'associazione o un gruppo di interesse di essere influente e di «contare» dipende dalle risorse di scambio che possiede e dalla legittimazione sociale che riesce a riscuotere giocando sulla sua rappresentatività (chi, quanti, dove sono i soggetti che rappresenta), la sua legittimazione interna (la capacità di imporre comportamenti e scelte ai soggetti rappresentati), la sua competenza (la capacità di risolvere tecnicamente i problemi). Il meccanismo del collateralismo svalutava l'importanza di tali risorse rispetto all'identificazione politica ed impostava lo scambio - ecco la pervasività sociale - sul controllo e sulla «manutenzione» del consenso: la rappresentatività veniva stemperata all'interno della galassia degli interessi funzionali collaterali al partito per il quale era più rilevante la fedeltà; la capacità di imporre scelte e comportamenti era in buona misura delegata al partito stesso ed al suo controllo gerarchico ed espressa più in termini di adesione ideologica ad una delle due «chiese» che di identità o meccanismi organizzativi dell'associazione; la competenza tecnica era comunque risorsa minimale rispetto alla capacità di scambiare benefici col consenso politico. 
Le contraddizioni della transizione e l'implausibilità di un neocollateralismo

La transizione di regime che ha cominciato a caratterizzare il nostro sistema politico all'inizio degli anni ' 90 , se esaminata dall'angolo di visuale che qui ci interessa, rivela alcune dimensioni interessanti.

Il nodo centrale può essere così sinteticamente espresso: se la transizione deve essere considerata anche come un processo di ridefinizione delle identità collettive, la minore capacità di integrazione e regolazione dei partiti politici ha aperto una sorta di «vuoto» che altri attori istituzionali tra i quali anche le associazioni di rappresentanza, con le proprie risorse di legittimazione, hanno potuto potenzialmente occupare e colmare. Tale tesi comporta anche un ovvio carattere limitativo: rilevare che la transizione ha aperto maggiori spazi di azione alle associazioni non vuol dire ipostatizzare tale fenomeno e concepirlo come assetto strutturale compiuto o definitivo. Di transizione infatti si tratta, ed il processo di ridefinizione delle identità collettive non può certo assegnare ai partiti (o ad altre forme di azione collettiva come i movimenti single-issue) un ruolo indefinitamente passivo. Ci limitiamo a prendere atto che la transizione non sembra aver scalfito le capacità aggregative delle associazio$\mathrm{ni}$ - come dati di tesseramento, sondaggi di gradimento e ricerche ad hoc sembrano ampiamente confermare ${ }^{12}$ - e ha reso tali attori più rilevanti di quanto siano stati in precedenza. Ecco quindi che la crisi dei modelli di regolazione e dei meccanismi di interdipendenza tra sistema partitico e sistema associativo propri del recente passato ha generato la necessità, da parte del sistema della rappresentanza imprenditoriale, di ridiscutere le proprie logiche di azione al fine di ristabilire fondamentali principi tanto di coerenza con le logiche del processo decisionale pubblico quanto di efficacia nel perseguimento dei propri fini: coerenza ed efficacia oggi non più garantite da quel particolare modello di collateralismo che abbiamo descritto. $\grave{E}$ in questo

12 Una ricerca curata da Diamanti e dall'Istituto Poster nel 1995 in provincia di Vicenza sul grado di affidabilità delle istituzioni locali percepito dai cittadini, ad esempio, ha visto piazzarsi al primo posto, davanti alla Chiesa, nientemeno che l'Associazione degli Artigiani ed al terzo l'Associazione degli Industriali. Pur senza raggiungere esiti così clamorosi anche le già citate ricerche condotte dall'Osservatorio dell'Università di Bologna confermano le crescente legittimazione sociale di molte associazioni imprenditoriali. 
senso che l'impatto della transizione di regime sulla rappresentanza imprenditoriale italiana si fonda sulla discrepanza tra opportunità innovative ed impossibilità dei meccanismi storicamente più collaudati a coglierle. La questione centrale è, dunque, in che misura la transizione di regime ha incrinato il modello pre-esistente di rapporto tra partiti ed associazioni.

Sul piano logico vi sono almeno quattro nitidi fattori che non solo hanno causato la crisi di quel tipo di collateralismo, ma che dovrebbero anche renderne implausibile la restaurazione. I primi tre sono sufficientemente ovvi. In primo luogo, la rottura dell'unità politica dei cattolici ha privato una parte delle associazioni dello storico e consolidato referente politico, la cui scissione organizzativa non permette di discriminare tra gli schieramenti politici oggi contrapposti. In secondo luogo, la nuova legge elettorale, nella sua parte maggioritaria, si fonda su collegi di piccole dimensioni al cui interno nessuna delle attuali associazioni può contare al punto da determinare il successo di un candidato proprio o «sponsorizzato». Sono le stesse regole del gioco ad inibire la possibilità di un eventuale rapporto di scambio fondato sul consenso ${ }^{13}$. In terzo luogo, la logica della legge elettorale rende estremamente rischiosa per qualsiasi associazione una scelta preventiva di schieramento politico, non foss'altro perché ad urne chiuse sarebbe ben chiaro chi sosterrebbe per la durata della legislatura i costi di una difficile convivenza con un governo «nemico».

A questi tre fattori ne va aggiunto un quarto: il collateralismo non è più un modello efficiente perché sono venute meno tanto le logiche claniche fondate sulle forti identità collettive,

13 Roberto Cartocci mi ha fatto notare che a suo parere il maggioritario non diminuisce l'influenza delle associazioni in quanto l'impegno di un'associazione, concentrato in pochi collegi incerti, può essere decisivo per la vittoria di alcuni candidati da collocare poi nelle commissioni parlamentari. L'obiezione è teoricamente plausibile ma non del tutto convincente. Anzitutto è evidente che, rispetto al sistema elettorale proporzionale, la diminuzione dell'ampiezza dei collegi va tutta a svantaggio delle associazioni che, nel calcolo della concentrazione dei (davvero teorici) voti potenziali, incidono assai meno che in passato. Se vogliamo invece ragionare esclusivamente sui collegi marginali, allora è davvero improbabile che nei collegi ad alta incertezza sia proposto come candidato qualcuno identificabile con uno specifico gruppo di interesse: ciò diminuirebbe le sue possibilità di vittoria! Altra cosa, infine, è riconoscere che nei collegi marginali la capacità di pressione di ciascun singolo gruppo aumenta di pari passo con l'incertezza sugli esiti del voto: ma ciò non può che esaltare la capacità di mediazione del candidato tra i diversi interessi. Per questi motivi la diminuzione di influenza diretta sul voto che il sistema maggioritario concede alle associazioni, rispetto a quello proporzionale, $\mathrm{mi}$ sembra indiscutibile. 
quanto le capacità gerarchiche dei partiti nei confronti delle varie associazioni, non più in grado di integrare a livello sistemico le identità verticali. Da un lato, la crisi dei partiti è anche una crisi organizzativa di risorse economiche e materiali e quindi di capacità di presidio, indirizzo e controllo; dall'altro, la crisi delle grandi ideologie e la crescente mobilità di voto degli elettori hanno sottratto alle forze - oggi solo presuntivamente o nominalisticamente collaterali - il controllo del comportamento elettorale dei propri iscritti: il fenomeno della destrutturazione del mercato elettorale è ormai visibile (e rilevato dagli studiosi da vari $\left.a_{n n i}{ }^{14}\right)$ e si può sostenere che il suo impatto devastante sui meccanismi del collateralismo sia diventato immediatamente evidente dopo le elezioni del 21 aprile 1996, quando le scelte di schieramento dei sindacati confederali a fianco dell'Ulivo (pari a più di otto milioni di voti presunti) non è stato sufficiente ad evitare che il Polo conquistasse, se pur di poco, la maggioranza dei voti.

Di conseguenza, l'implausibilità di un neo-collateralismo deriva dal venir meno, al tempo stesso, delle regole del gioco favorevoli allo scambio previsto dal modello, della convenienza certa dello scambio, della stessa materia di scambio tra partito ed associazione ${ }^{15}$. La transizione ha dunque un impatto quasi paradossale sul sistema associativo: mentre offre spazi di azione

14 Cfr. ad esempio: Manneheimer e Sani (1987), Cartocci (1990), Sani (1992), Diamanti e Mannheimer (1994).

15 Tale analisi si pone in parziale contraddizione con altre recenti interpretazioni dell'evoluzione del rapporto tra partiti e gruppi di interesse. In particolare Mattina (1994a, 1994b), analizzando il comportamento elettorale dei gruppi di pressione nel '94, sostiene che la loro mancata mobilitazione in termini di candidature, espressione di un progressivo declino del loro impegno diretto, è il risultato di una serie di cause tra cui la crescente professionalizzazione del ceto parlamentare di estrazione partitica, la crescente crisi di rappresentatività di diverse associazioni (tra cui principalmente $\mathrm{i}$ sindacati), la difficoltà di azione unitaria definita dai centri confederali. A mio parere, le cause della mancata mobilitazione vanno invece ricercate anzitutto nella crisi del collateralismo così come l'abbiamo sopra analizzata; a ciò aggiungerei che: a) non è generalizzabile, né condivisibile, la diagnosi di crisi di rappresentatività delle associazioni, perché se è vero che i sindacati aumentano gli iscritti solo colmando il turn-over con i pensionati, questo è un fenomeno che non tocca la maggior parte delle associazioni imprenditoriali, alcune delle quali, come si è detto, hanno negli ultimi anni costantemente aumentato i propri iscritti ed ampliato la propria legittimazione interna ed esterna; $b$ ) concordo con le caratteristiche di «sistema a legame debole» delle associazioni (sul punto rinvio al penultimo paragrafo), ma questo aspetto non è particolarmente rilevante in relazione all'attuale sistema elettorale in quanto il meccanismo del recupero proporzionale ha spostato i giochi su liste e candidature dai collegi alle sedi romane dei partiti ed è a questo livello centralizzato che le associazioni hanno giocato, tagliando completamente fuori le periferie. 
storicamente inusitati, scardina, al tempo stesso, i meccanismi più consolidati. Ciò è ben visibile da un esame delle dinamiche più recenti.

\section{Le associazioni e le elezioni del 1996}

Il comportamento pre-elettorale delle associazioni imprenditoriali risulterà più evidente se guardiamo alle alternative in quel momento disponibili. In questo modo emergerà anche una netta differenza di comportamento tra Confindustria, sindacati confederali e le altre associazioni imprenditoriali. Rispetto a più precise tipologie del rapporto tra gruppi di interesse e partiti ${ }^{16}$, preferisco qui sintetizzare le alternative in tre semplici ipotesi di fondo. Della prima ipotesi, fondata su una scelta di affiancamento (partigiano e preventivo) ad un partito o ad una coalizione, abbiamo già detto. La seconda ipotesi consiste nel ridefinire il collateralismo sulla base di un processo di identificazione degli «interessi naturali» (Feltrin e Zan 1995) rappresentati dall'impresa e dai lavoratori, e implica uno schierarsi quasi obbligato, e perciò «naturale», del Lavoro con la sinistra e del Capitale con la destra. Tale ipotesi, verosimile soprattutto sulla base di una valutazione storica ed empirica degli assetti associativi nelle democrazie avanzate, presenta tuttavia alcuni limiti evidenti: in primo luogo, costituisce solo una variazione del collateralismo, in cui la dinamica portante è data non più dallo scambio politico-elettorale ma dalla coincidenza (tendenziale e prevalente) di interessi sociali ed interessi funzionali; in secondo luogo, ipostatizza la dicotomia tra destra e sinistra sulla base del modo con cui queste due categorie sono tradizionalmente e storicamente intese e senza tener conto dell'ampio e problematico

16 Morlino (1991) costruisce un continuum ai cui estremi pone, da un lato, l'assetto in cui i partiti fungono da «controllori d'accesso» (gatekeepers) rispetto agli interessi sostenuti dai gruppi (ossia in cui i gruppi hanno necessità dell'intermediazione partitica per l'accesso alle arene decisionali), e, dall'altro, la situazione opposta di accesso diretto da parte dei gruppi al decisore pubblico e di posizione secondaria dei partiti. Tra i due estremi si colloca una serie di situazioni intermedie che ricomprendono tre ipotesi: il dominio condizionato del partito sul gruppo, la simbiosi tra partito e gruppo, la neutralità del gruppo rispetto al partito. Due ragioni mi hanno indotto a una semplificazione delle ipotesi: assumere come prospettiva la dicotomia, centrale nel momento politico in questione, tra identificazione e non identificazione partitica cui si sono trovate di fronte le singole associazioni; dominio condizionato e simbiosi sono comunque articolazioni di differente intensità dell'ipotesi collateralista. 
dibattito sulla loro ridefinizione negli attuali contesti socio-economici; in terzo luogo, forzando l'ipotesi della naturalità degli interessi, si pone in contraddizione con la componente non ideologizzata e mobile del comportamento di voto. La terza ipotesi consiste nel proporre un nuovo modello in chiave di «autonomia associativa», $\mathrm{i}$ cui assunti sono l'equidistanza dell'associazione dagli schieramenti in campagna elettorale e la successiva contrattazione del consenso con le differenti componenti partitiche o coalizionali di volta in volta legata ai singoli provvedimenti. Anche in questo caso sono necessarie alcune precisazioni, anzitutto sull'accezione di autonomia. Occorre, ad esempio, distinguere tra autonomia come esito di una scelta di non schieramento politico e autonomia come efficacia dell'azione di rappresentanza, giacché non è scontato che l'una implichi necessariamente l'altra. Avendo come oggetto di analisi il sindacato dei lavoratori, Feltrin (1994) postula che esso possa dichiararsi autonomo quando si verificano due condizioni: a) che sia forte, in quanto la debolezza è per definizione mancanza di autonomia; $b$ ) quando misura i suoi comportamenti contrattuali sulla base degli interessi specifici che rappresenta e non di quelli dell'arena più ampia in cui agisce. Se è così, allora si può dare il caso di un sindacato politicamente schierato, ed anche molto esposto, eppure sufficientemente forte da essere autonomo (è il caso della Germania).

Se applichiamo questo schema alle associazioni datoriali italiane - consci del fatto che in un sistema bipolare esse non sono affatto speculari al sindacato, che può essere più o meno autonomo solo dal versante politico pro-labour -, possiamo osservare che l'attuale frammentazione della rappresentanza delle singole categorie rende le associazioni (tutte ad eccezione della Confindustria) soggetti non ancora sufficientemente forti. Col che la pretesa di autonomia assume i tratti del velleitarismo. Infatti nel momento in cui ciascun settore produttivo si presenta con una pluralità di istanze rappresentative, però non legittimate a rappresentarlo integralmente, da un lato scaturisce inevitabilmente - con il gioco di sponde e di veti - un'interazione tra le differenti associazioni simile a quella celebre tra i capponi di Renzo Tramaglino, dall'altro l'opportunismo del decisore pubblico nel definire le scelte sarà sollecitato per eccesso ${ }^{17}$. A dimostrazione di

17 È esattamente a quest'ultimo aspetto che Prodi ha più volte fatto riferimento incontrando le associazioni di categoria nel corso della campagna elettorale. 
ciò sta l'influenza che hanno esercitato sindacati confederali e Confindustria sulle più importanti scelte di governo compiute negli ultimi anni (dall'accordo sul costo del lavoro alla riforma del sistema pensionistico alle leggi finanziarie) rispetto al più basso profilo sostanziale tenuto dalle altre associazioni.

L'esame delle alternative - ognuna delle quali, sia pure per motivi diversi, non appare allo stato attuale plausibile o perseguibile - rende maggiormente comprensibile l'effetto della transizione dal punto di vista della rappresentanza funzionale e ne costituisce un diretto indicatore. Esso si sostanzia poi nelle scelte concrete compiute dalle associazioni in campagna elettorale. Infatti soltanto Confindustria e sindacati sono stati in grado di abbracciare con chiarezza una delle tre opzioni: da un lato, i sindacati hanno proseguito senza troppi tentennamenti sulla via già esperita con l'opposizione al governo Berlusconi e durante la campagna elettorale hanno dato indicazione di voto a favore del centro-sinistra, nonostante una parte dei loro iscritti (come abbiamo già detto) sia orientata in maniera opposta; dall'altro, la Confindustria ha ribadito la sua posizione «apartitica e agovernativa» ed è stata molto attenta nel confronto con i candidati a correggere via via l'impressione che fosse maggiormente favorevole all'una o all'altra parte.

Quasi tutte le altre associazioni imprenditoriali hanno mostrato, al contrario, una certa dose di schizofrenia pendolando tra l'una e l'altra opzione, senza abbracciarne alcuna, senza precludersi la strada verso nessuna: frenate dall'eterogeneità degli schieramenti e dall'incertezza sugli esiti (con l'eccezione di Confagricoltura, che ha eletto alcuni parlamentari in Forza Italia, e della Lega delle cooperative che ha eletto il suo presidente con l'Ulivo ${ }^{18}$ ). Le associazioni una volta definite «rosse» erano infatti imbarazzate tanto da una sorta di schieramento «derivato» con i sindacati quanto da un collegamento culturalmente e politicamente difficoltoso col centro-destra (ad esempio Cia e Confesercenti, pur più vicine all'Ulivo, non hanno presentato candidati nelle sue liste), mentre le associazioni «bianche», ten-

18 La Confagricoltura, storicamente vicina al Partito liberale e alla destra Dc, come espressione della grande proprietà agraria si è venuta a trovare in una posizione di crescente isolamento sia (per ragioni di membership) dalla Coldiretti che dalla Cia (per ragioni di cultura politica); la Lega delle cooperative - cito le parole di un dirigente - «ha fatto di necessità virtù rispetto all'ostilità dichiarata di Berlusconi». Di qui la scelta collateralista delle due associazioni. 
denzialmente più inclini all'ipotesi degli interessi naturali, e quindi più portate per il Polo, si trovavano alle prese con interlocutori storicamente non consolidati e con referenti dispersi fra i due schieramenti. Il comportamento di fatto prevalente è stato quello di un'autonomia troppo spesso ribadita nelle parole e poco consistente nella pratica, e nella scelta assai più tattica che strategica di un opportunismo politico inteso come inevitabile schieramento (ex post) col vincitore della contesa elettorale, ragion per cui ad urne chiuse tutti i principali dirigenti associativi si sono scoperti e dichiarati «filogovernativi». In realtà ad emergere con chiarezza è stata solo la persistenza, o meglio ancora, la «vischiosità» dei meccanismi del tradizionale collateralismo. Tale persistenza può essere sinteticamente ricondotta a cinque ragioni.

La prima ragione è la più ovvia. La fluidità e l'incertezza che hanno caratterizzato lo scenario politico negli ultimi anni, la campagna elettorale continuativa, il gioco al rinvio tra elezioni e riforme, la sfiducia che le elezioni fossero in grado di partorire una maggioranza di governo, l'incertezza sul possibile vincitore delle stesse, sono tutti elementi che hanno contribuito a congelare ogni iniziativa di carattere radicalmente innovativo.

$\mathrm{La}$ seconda ragione fa ancora riferimento al meccanismo elettorale nella sua «imperfezione». Su una cosa osservatori e parti in causa sono unanimemente d'accordo: l'attuale legge elettorale addirittura esalta, rispetto al passato, il ruolo oligarchico delle segreterie nazionali di partito nella definizione delle liste elettorali, in contraddizione con lo spirito maggioritario del radicamento territoriale dei candidati. $\mathrm{Ma}$ anche se i giochi vengono giocati soltanto a Roma, anziché nei collegi, ciò non implica vantaggi per le associazioni che anzi, orfane della mediazione partitica e senza il surrogato di un reale potere contrattuale, oggi possono ottenerli soltanto rischiando e «sovra-esponendosi» politicamente, ossia grazie ad una scelta di schieramento.

La terza ragione è più complessa e può essere definita come eccesso di domanda di rappresentanza. Possiamo esemplificarla laddove è più visibile, il Nord-Nord-est del paese, utilizzando il voto leghista come una sorta di cartina di tornasole. Tra le analisi del voto possiamo richiamarci a Diamanti (1996a, 1996b) che rileva due aspetti particolarmente interessanti: il primo consiste nel fatto che il voto leghista, ben lungi dall'essere esclusivamente una manifestazione unanime di istanze autonomiste, è assai più un voto di protesta legato a fattori prevalentemente 
socio-economici o un voto di attesa e non scelta tra le due maggiori coalizioni in campo; il secondo aspetto è legato alla sua distribuzione territoriale che coincide, in tutta la fascia subalpina e perfino nell'enclave cuneese, con la maggiore densità di voti Dc nelle elezioni del '48. Diamanti argomenta che esiste in queste aree una visibile continuità di domanda politica una volta saturata dalla Dc e poi, dall'inizio degli anni ' 80 , con la crisi del sistema del notabilato territoriale come meccanismo di raccolta e canalizzazione di benefici e con la conseguente altissima incertezza dell'elettorato - incertezza maggiore in quanto proveniente da territori caratterizzati da forte espansione economica - a poco a poco conquistata dalla Lega. Il voto leghista, secondo Diamanti, è dunque fondamentalmente un voto legato all'incertezza: incertezza della domanda politica tanto sulla collocazione politico-ideologica quanto sulla possibilità di far valere le proprie istanze sociali ed economiche, incertezza dell'offerta politica nel cogliere le determinanti reali della richiesta di rappresentanza: è su queste basi che si colloca il consenso della Lega e «precipita», sul piano simbolico, la dimensione di protesta. Al di là delle ipotesi sulla continuità del voto Dc - Lega, ciò che qui interessa far notare è come il Nord-est, ormai ovunque celebrato per la sua elevatissima densità imprenditoriale $\mathrm{e}$ dove alcune associazioni ritrovano percentuali importanti della loro base associativa e delle loro entrate economiche, si ritrovi a soffrire di una carenza di offerta politica che sfocia in una straordinaria richiesta di rappresentanza. Ciò che ne scaturisce è una sorta di discrasia tra la scelta di votare Lega, enfatizzando la dimensione della rottura (con il sistema partitico, con Roma, con le prassi istituzionalizzate) e la necessità di appoggiarsi sempre più, sul piano pragmatico della reale tutela degli interessi funzionali, alle associazioni di rappresentanza. La conseguenza, ed in ciò sta la discrasia, è che le associazioni si ritrovano imbrigliate tra una spinta ad una sorta di «collateralismo forzato» - con una sponda politica che non è però interessata a rivestire il ruolo che in passato fu della Dc - ed il pericolo di perdere legittimazione e consenso, con il risultato (solo apparentemente paradossale) che il processo di rafforzamento dell'identità e della rappresentatività delle categorie imprenditoriali è tanto più difficoltoso quanto meno sono presenti interlocutori politici di riferimento e quanto più di converso cresce la domanda di rappresentanza. Ecco quindi come il voto leghista può fungere da cartina di tornasole nell'analisi dei sistemi asso- 
ciativi, nel senso che rende maggiormente visibili ed enfatizzati, su una scala territoriale limitata, processi che hanno una valenza ed un respiro nazionali. La perdita, per tutte quelle associazioni imprenditoriali che in passato costituivano la galassia di riferimento funzionale della Dc, del referente politico ed al contempo la crescita della domanda di rappresentanza rendono più difficoltosa sia la definizione dell'identità associativa sia la scelta dell'autonomia politica ed incentivano anzi la tentazione di schieramento (con il centro-destra) che, proprio nella maggiore domanda di rappresentanza che viene dal basso, trova forse non legittimazione, ma quanto meno un potente propellente.

La quarta ragione della persistenza collateralista può ricondursi a quella precedente ricomprendendo nell'analisi anche le associazioni di versante Pds. L'autonomia è evidentemente un problema di relazione che si pone nei confronti degli «amici» (le forze politiche di riferimento) e che non sussiste nei confronti dei «nemici» da cui si è sempre autonomi (Feltrin 1994). Di conseguenza l'autonomia costituisce un problema «strutturale» per le associazioni «rosse» mentre sembrerebbe un problema assai più virtuale per quelle «bianche». In effetti le associazioni di versante «rosso» sembrano oggi assai più tese a puntualizzare e a distinguere la propria posizione rispetto agli schieramenti politici, pena il rischio reale di una perdita di iscritti; al contrario le associazioni di versante «bianco», che non sono costrette ipso facto all'autonomia ma che non costituiscono da sole soggetti sufficientemente forti, sono costrette a sviluppare strategie che presuppongono minore autonomia politica. Dal punto di vista sistemico si tratta di un effetto quasi paradossale o «perverso» del genere «dilemma del prigioniero» (Elster 1979), dettato dalle regole del gioco ed imperniato su valori profondamente radicati nelle varie organizzazioni, culturalmente incapaci di uscire da un gioco che ha come pegno la difesa dalla gerarchia partitica e come posta la possibilità di avvalersene.

La quinta ed ultima ragione non può trascurare l'impatto che tuttora esercita la cultura politica delle élites e dei gruppi dirigenti associativi, che sono stati selezionati, formati e cooptati nelle oligarchie partitiche condividendone scelte e strumenti di attuazione secondo la logica dei «direttorati incrociati», e le cui risorse personali continuano ad essere composte di valori, capacità, relazioni profondamente radicati nel passato e assai poco permeabili agli stimoli ed ai rischi del nuovo.

A questo punto, traendo delle sintetiche conclusioni dalla 
disamina del comportamento pre e post-elettorale delle associazioni imprenditoriali, potremmo sostenere che la situazione attuale si caratterizza più che altro come fase di stallo e di attesa rispetto alle più ampie dinamiche politiche ed istituzionali. Una tale diagnosi, tuttavia, non coglierebbe l'effetto più rilevante della transizione su tali associazioni: il processo di ridefinizione di confini, strutture ed identità delle diverse associazioni imprenditoriali. Che tale processo sia effettivamente avviato è dimostrato da una pluralità di casi (monitorati dall'Osservatorio sulle Associazioni di Rappresentanza dell'Università di Bologna) relativi a diverse associazioni: l'Associazione Industriali e l'Associazione Piccola Industria si sono unificate a Treviso e a $\mathrm{Pa}$ dova, Confartigianato e Cna a Lecco e a Livorno; Cna e Confeserecenti stanno per unificare le società di servizi a Modena, la stessa cosa è già stata fatta da Confartigianato e Cna ad Arezzo; Confcommercio, Coldiretti e Confartigianato hanno siglato formali ed inediti protocolli d'intesa a livello regionale e nazionale; infine, a livello nazionale, in varie associazioni si ragiona ormai apertamente di opportunità e modalità di unificazione, confederazione, unità organica, patto di sindacato e così via ${ }^{19}$. La questione centrale ha, dunque, ormai oltrepassato la logica del rapporto col sistema dei partiti e coinvolge la complessiva ridefinizione del sistema della rappresentanza imprenditoriale italiana al fine di aumentare le possibilità di influenza delle diverse categorie rispetto alle due parti sociali più forti e consolidate, Confindustria da un lato e sindacati confederali dall'altro. Per comprendere però quali siano le attuali coordinate evolutive e per poter formulare alcune ipotesi interpretative non è sufficiente - e sarebbe deterministico e riduttivo ${ }^{20}$ - riferirsi a varia-

19 Nel settembre '96 si è tenuto a Riccione un seminario che ha coinvolto i gruppi dirigenti di Cna, Confartigianato e Casa e che ha fatto seguito ad un'analoga iniziativa tenutasi a Cortona nel settembre dell'anno precedente. Scopo del lavoro è stato la definizione di una «Carta dei valori» su cui aggregare le istanze delle tre Confederazioni e la messa a punto di un protocollo d'intesa per la costituzione di una Confederazione Unitaria dell'Artigianato che ha inserito formalmente nelle agende la questione. L'aver partecipato a tali seminari (assieme a Paolo Feltrin e a Stefano Zan, con i quali condivido questo filone di ricerca e di riflessione) non è certo stato irrilevante per la messa a fuoco di alcuni dei ragionamenti presentati in questo saggio.

20 Il mio intento è di non aderire ad una visione deterministica della transizione di regime e del rapporto tra arena politica e gruppi di interesse sulla scia, ad esempio, di modelli interpretativi come la «teoria delle contingenze» (Lawrence e Lorsch, 1967). Al contrario la mia impostazione è quella della «teoria dell'attore sociale», esposta tra gli altri da Crozier e Friedberg (1976) che formulano anche la miglior critica disponibile delle teorie contingentiste. 
bili esogene e contestuali, ma occorre tenere conto di dimensioni di carattere strutturale e culturale interne alle associazioni.

\section{Le associazioni imprenditoriali come attori organizzativi}

La letteratura politologica non si è quasi mai occupata approfonditamente dei gruppi di interesse in quanto organizzazioni caratterizzate da dinamiche proprie e peculiari rispetto ad altri tipi di organizzazione (gli stessi partiti politici, ad esempio). La scarsa conoscenza di questi fenomeni ha perciò generato una sorta di duplice «pregiudizio»: da un lato, una visione interpretativa del comportamento dei gruppi di interesse quasi esclusivamente limitata al loro punto di massima visibilità, che in genere coincide con un gruppo dirigente nazionale e con le sue scelte, strategie, dichiarazioni; dall'altro lato, una visione interpretativa più ampia rispetto all'insieme delle varie componenti strutturali dell'organizzazione e tuttavia tesa ad evidenziarne e a coglierne gli aspetti formali (sostanziabili prevalentemente negli organigrammi e negli statuti). Nell'uno come nell'altro caso vengono a mancare le basi necessarie alla più piena comprensione. In questa sede, e a partire dalla letteratura significativa comunque esistente ${ }^{21}$, mi limiterò a richiamare brevemente solo tre dimensioni rilevanti per la prospettiva che ho adottato: la gerarchia nominale, l'identità debole, il fattore servizi. Queste tre dimensioni mi sembrano sufficienti a dar conto dei vincoli che, al di là delle scelte politiche, rendono molto più difficile il perseguimento e la messa in opera di strategie realmente innovative quando esse coinvolgono, in ciascuna associazione, gruppi dirigenti vasti ed eterogenei ed apparati composti da migliaia di funzionari. In molti casi i comportamenti associativi possono essere meglio spiegati sulla base delle loro dinamiche organizzative interne più che a partire dal contesto politico ed istituzionale esterno.

Per quanto attiene la prima dimensione, occorre ricordare con Zan (1992) che le associazioni sono «sistemi a legame debole», ossia sono costituite da una pluralità di sottosistemi (di

21 Tra le eccezioni alle suddette visioni ricordiamo almeno: Regini (1981) per l'analisi del sindacato, Lanzalaco (1990) per l'analisi della Confindustria, Zan (1992) per la teorizzazione delle associazioni di rappresentanza come attori organizzativi. Per un'analisi, anche organizzativa, dei partiti il riferimento rimane Panebianco (1982). 
carattere orizzontale o verticale) dotati di propria sovranità, autonomia nonché delle risorse economiche necessarie, tant'è che i vertici nazionali funzionano grazie alle risorse loro cedute dalle periferie e non viceversa. Pertanto la raffigurazione grafica classica che si fa delle associazioni, e che passa per la scansione dei livelli nazionali, regionali e provinciali, configura un assetto presuntamente gerarchico ma di fatto puramente nominalistico; la stessa ripartizione di ruoli, funzioni e poteri che è dettata dagli statuti è per lo più ipotetica e non lascia strumenti reali di controllo e sanzione al vertice nazionale. In molte associazioni abbiamo osservato delle articolazioni mettere in atto comportamenti clamorosamente antistatutari (ad esempio il mancato versamento del contributo sul tesseramento) che non sono sanzionati né sanzionabili in quanto l'unica sanzione possibile, l'espulsione, non può essere attuata perché implica per definizione l'indebolimento dell'associazione.

Le associazioni non soltanto vivono all'interno di arene politiche ma costituiscono esse stesse vivaci arene politiche. Si può ben comprendere, pertanto, come gli assetti strutturali esprimano di per sé una forza inerziale ed un conseguente alto tasso di conservatorismo, derivanti dal prevalere di una pluralità di razionalità locali rispetto ad una ipotetica razionalità globale (Elster 1979). In tali assetti una qualsiasi scelta di ampia portata deve passare per una faticosa costruzione del consenso e, in ogni caso, vi è sempre la concreta possibilità di provocare spaccature insanabili, fuoriuscite di iscritti, diaspore, ossia di ritrovarsi alla fine del processo con l'associazione indebolita. Al contrario, le articolazioni locali hanno molte maggiori possibilità di manovra in quanto in ciascun contesto - a seconda delle caratteristiche sociali, economiche, politiche prevalenti - possono godere di elevati differenziali di potere rispetto ad altre categorie e dare luogo a scelte di cooperazione o conflitto assai più incisive sul loro ambiente di quanto sia concesso ai livelli nazionali. Ciò concorre, ad esempio, a spiegare i casi di unificazioni concluse o in corso (a Padova, Treviso, Arezzo, e così via) che abbiamo precedentemente citato e la cui complessiva incoerenza (strutturale o politica) sarebbe altrimenti misteriosa. Ciò spiega, infine, perché le dinamiche delle associazioni imprenditoriali che scaturiscono dalla transizione abbiano ritrovato finora maggiore visibilità esterna a livello locale anziché a livello centrale.

La seconda dimensione è quella dell'identità. In letteratura 
si è ampiamente d'accordo nel descrivere le associazioni di rappresentanza come organizzazioni a forte grado di istituzionalizzazione (Lanzalaco 1990; Zan 1992). Ciò che «tiene assieme» il sistema a legame debole associativo è costituito, da questo punto di vista, da tutti quei fattori di carattere culturale a partire dai quali si costruisce e si determina l'identità di un'organizzazione a base volontaria. Il processo attraverso cui ciò avviene è per l'appunto l'istituzionalizzazione (Selznick 1957), ossia quel processo in base al quale l'organizzazione perde il suo carattere strettamente strumentale per divenire un valore in sé, qualcosa che prescinde dai livelli di efficacia e di efficienza che è in grado di esprimere. Ogni istituzione, per definizione, è rigida e poco disponibile a cambiamenti che mettano in discussione valori, principi organizzativi, routine consolidate: anche il guardare alle associazioni come istituzioni fornisce un ulteriore argomento per rendere conto della loro difficoltà nei confronti dell'innovazione. Tuttavia è necessario approfondire questa dimensione mettendo a fuoco due elementi: l'istituzionalizzazione delle associazioni imprenditoriali è in realtà meno forte di quanto si sarebbe portati a credere; tali associazioni sono caratterizzate da un'identità che non esiterei a definire «debole».

Il primo elemento attiene alla sfera dei valori, il secondo a quella degli interessi. Partiamo dai valori: come abbiamo già ampiamente sottolineato il meccanismo del collateralismo politico si è fondato su di un'ampia delega da parte delle associazioni al partito di riferimento ed alla sua ideologia, anche in tema di valori, di pedagogia, di integrazione sociale, ragion per cui anche l'identità associativa era prevalentemente «proiettata» sull'associazione dall'esterno e dai fondamentali assunti ideologici definiti dal partito. In ciò non posso non concordare con Pizzorno (1993) quando afferma che meccanismi di tal fatta producono due esiti: l'indebolimento dei principi su cui si fonda la coesistenza pluralistica e l'irrigidimento su principi ideologici dei criteri di selezione del personale dirigente. Questi esiti si traducono anche nella bassa capacità di mobilitazione sociale su presupposti non ideologici o nell'esclusione di chi a tali presupposti non aderisce. Sul versante della rappresentanza funzionale, ciò è stato tanto più vero per le associazioni imprenditoriali - alle quali è sempre mancato quel contrappeso identificativo ed assiologico interno che hanno avuto invece i sindacati con il movimento operaio (ed infatti il pro- 
blema dell'autonomia dei sindacati dai partiti si è posto con vent'anni di anticipo) - e in qualche misura anche per la Confindustria, con la mitizzazione ideologica della grande impresa (come modello socialmente auspicabile per la sua razionalità e la sua efficienza, da proporre come paradigma: l'azienda Italia, l'azienda ospedale, e così via).

La mancanza di un sufficiente sostrato di valori identificativi propri delle associazioni imprenditoriali potrebbe tuttavia essere bilanciata da un forte elemento strutturale, ossia la forte omogeneità degli interessi espressi, dimodoché il processo di definizione dell'identità del gruppo sarebbe ricondotto alla sua natura più «tecnica»: la definizione dei confini organizzativi e dei criteri di inclusione e di esclusione fondati sulla natura degli interessi rappresentati e rappresentabili. Ma anche da questo punto di vista molte associazioni imprenditoriali manifestano debolezza. Molte categorie economiche, cui oggi corrispondono specifiche associazioni di rappresentanza (sicuramente gli artigiani, i commercianti, le Pmi, in qualche misura anche gli agricoltori), ricomprendono infatti al loro interno una pluralità di mestieri e di attività economiche, e quindi di interessi specifici: ad esempio il tassista, l'idraulico ed il contoterzista nelle associazioni dell'artigianato; il piccolo dettagliante, l'albergatore e il grande distributore in Confcommercio. Di tale confusa logica della membership è esempio ulteriore che un'associazione di artigiani come la Cna voglia, statutariamente, rappresentare anche gli interessi delle piccole e medie imprese in concorrenza con la Confapi (e con la Confindustria!) e ricompren$\mathrm{da}$ tra $\mathrm{i}$ soci anche un numero non esiguo di commercianti. L'accentuazione quantitativa della membership rende quindi più difficoltosa la valorizzazione dell'influenza di ciascuna associazione e la debolezza dell'identità si sposta dal piano strutturale - della composizione degli interessi rappresentati - al piano soggettivo e strumentale dell'ampliamento degli interessi potenzialmente rappresentabili. Come si è già detto, tutte queste associazioni, a seguito della dissolvenza ideologica, hanno accentuato negli ultimi anni la mobilità e l'inclusività dei propri confini (competendo tra loro sul tesseramento, anche tra categorie non omogenee; cercando di affiliare ogni piccola associazione verticale non aderente ad alcuna centrale confederale). La tendenziale frammentazione della membership rende così puramente nominalistico l'aumento di influenza che do- 
vrebbe derivarne e ha come unica conseguenza un ulteriore indebolimento di identità 22 .

Il rischio dell'erosione del «collante associativo» (Zan 1992) connesso a un'identità debole - in quanto eccessivamente sfumata, disarticolata, differenziata, mobile - comporta un'ulteriore conseguenza: la necessità per tali associazioni di offrire ai propri associati ragioni di scambio valutabili in termini tendenzialmente «istantanei», ossia di breve periodo, come è facilmente comprensibile alla luce della teorizzazione dei costi di transazione. Infatti, laddove vigono logiche claniche (vi è condivisione di valori comuni, vi è identità di gruppo, vige lo «spirito comunitario» weberiano, prevale la fiducia reciproca) l'equità delle ragioni di scambio tende ad essere differita nel tempo: essa, cioè, diviene «seriale» (Ouchi 1980). Al contrario, la mancanza di tali condizioni anticipa la valutazione dell'equità degli scambi da parte dei soggetti sociali, fino al principio di istantaneità proprio del mercato (Williamson 1975). Ed è infatti un meccanismo di mercato che prevale tra la maggior parte delle associazioni imprenditoriali rispetto agli scambi con i propri associati: d'altronde la condizione di reciproca concorrenza sui medesimi segmenti di associati (conseguenza sia della duplicazione o triplicazione delle organizzazioni di rappresentanza in categorie omogenee, sia della concorrenza ormai anche intercategoriale) offre facilmente l'opzione di exit rispetto all'onere della voice che, come ricorda Hirschman (1970), scatta in condizioni di mercato monopolistico o per ragioni di loyalty verso l'organiz-

22 Si potrebbe argomentare che anche la Confindustria non sfugge ad un'eccessiva eterogeneità della membership, né ad una strategia di allargamento continuo dei confini. Si propone infatti come associazione di rappresentanza generale dell'impresa, ed al suo interno si ritrova la piccola accanto alla grande impresa, l'impresa pubblica o semi-pubblica accanto a quella privata, il grande produttore accanto al piccolo subfornitore, con un'evidentissima situazione di conflitto tra i differenti interessi. «L'integrazione della rappresentanza d'impresa», così definita da Abete, conta in effetti su due fattori: da un lato il suo carattere storicamente «filogovernativo» ma non collateralista, dall'altro la subalternità che alcune categorie economiche (soprattutto quelle di area Dc) hanno a lungo tollerato e coltivato. Di conseguenza la scelta confindustriale è stata di irrigidire in senso ulteriormente gerarchico uno storico rapporto di leadership nei confronti di alcune categorie produttive, scelta che oggi è però solo molto parzialmente concretizzata $e$ costituisce, dal mio punto di vista, un fattore di crisi di legittimazione rispetto al passato. Ma per i motivi ideologici sopra menzionati, per il peso storico della grande impresa privata (di cui la Confindustria ha il monopolio), per le risorse di potere che detiene, per la sua possibilità di influenza ed anche per «l'interdipendenza di privilegi» con i sindacati, non è possibile negare la supremazia confindustriale nel sistema della rappresentanza imprenditoriale, ciò che di per sé è ancora fonte di una capacità identificativa forte se comparata a quella delle altre associazioni imprenditoriali. 
zazione. Abbastanza diversa è la situazione della Confindustria, che monopolizza almeno la rappresentanza della grande impresa, e dei sindacati confederali, che sono sì scavalcabili da autonomi, Cobas e Gilde (un numero limitato dei quali, però, costituisce soggetto contrattuale, cioè istituzionalmente legittimato), ma dispongono pur tuttavia di meccanismi identificativi assai più forti sia in termini valoriali che strutturali. In ragione di ciò Confindustria e sindacati possono più facilmente imporre ai propri iscritti ragioni di scambio differite almeno nel medio periodo ed in tal modo spendere nell'arena politica la risorsa di legittimazione «interna» che possiedono e quindi aumentare sia la legittimazione esterna che la capacità di influenza sulle decisioni pubbliche ogni qual volta queste abbisognano di accordi o consenso con un raggio temporale non limitato ${ }^{23}$. Pertanto rileviamo che per le associazioni imprenditoriali di cui qui ci occupiamo l'identità costituisce un fattore di disgregazione associativa e di accentuazione della logica di membership: tale fattore calato in un meccanismo di competizione (tra le associazioni) e di scambio (tra ciascuna di esse e gli associati) che fa perno su opzioni di voice o di loyalty in minor misura di quanto non accada per gruppi di interesse maggiormente istituzionalizzati e meglio identificati - indebolisce la capacità di influenza e lo stesso processo di accreditamento sociale. Al tempo stesso rende più difficoltose scelte organizzative di ampio respiro strategico che possono contare meno sulla possibilità dell'associazione di gestire efficacemente il conflitto interno.

Veniamo infine alla terza ed ultima dimensione: il fattore servizi. Le associazioni imprenditoriali italiane si caratterizzano per un'ulteriore dimensione che non trova analogie sul piano quantitativo e qualitativo nemmeno con le omologhe associazioni di altri paesi europei: il fatto di aver sviluppato, in aggiunta all'attività di rappresentanza, un'attività di erogazione di servizi agli associati che coinvolge percentuali preponderanti dei loro

23 Fra i molti esempi possibili possiamo ricordare la prima tornata del luglio '91 dell'accordo sul costo del lavoro quando, in presenza di una fortissima opposizione della base della Cgil alla firma apposta da Trentin - e seguita dalle sue dimissioni -, una grande mobilitazione dei funzionari sindacali, condotta tramite assemblee fabbrica per fabbrica, fece alla fine scaturire il consenso della maggioranza della base quanto meno sull'inevitabilità dell'accordo. L'esempio è utile anche sul versante imprenditoriale: nel successivo luglio 1992 alcune associazioni (ad esempio Confartigianato e Confcommercio) rifiutarono di firmare contestualmente e lo fecero soltanto alcuni mesi dopo, quando l'accordo era ormai effettivo. 
organici e da cui deriva la maggior parte delle loro entrate economiche ${ }^{24}$. Questa dimensione ci consente di sviluppare alcune riflessioni e permette di articolare e precisare ulteriormente il ragionamento condotto nel paragrafo precedente in merito all'identità associativa e alle ragioni di scambio con gli associati. L'erogazione di servizi (amministrativi, gestionali, di consulenza, di formazione, di accesso al credito, e così via) indispensabili all'azienda costituisce infatti un potente fattore di stabilizzazione del rapporto con l'associato e di fidelizzazione dello stesso. Seguendo Williamson (1975) potremmo dire che le conoscenze «idiosincratiche» che nascono nel corso dello scambio irrigidiscono il rapporto tra l'erogatore e l'associato, rendendo il primo una risorsa altamente specifica, e dunque difficilmente sostituibile, per il secondo, al punto che interrompere il rapporto rivolgendosi alla concorrenza esistente sul mercato (altre associazioni o consulenti privati) diventa per l'associato estremamente costoso. Seguendo Olson (1965), d'altro canto, si potrebbe argomentare che rispetto alla rappresentanza di interessi che costituisce incentivo collettivo disponibile anche ai non iscritti e ai free-riders - l'erogazione di servizi funge da incentivo selettivo. In ogni caso, l'erogazione di servizi è una funzione associativa che, esigendo fiducia reciproca, rinsalda il legame con l'associato e ne aumenta il livello di «lealtà» nei confronti dell'organizzazione ${ }^{25}$. Non però senza conseguenze. Occorre in-

24 Nell'artigianato, settore sul quale abbiamo svolto ricerche empiriche più approfondite, le associazioni ci hanno fornito i seguenti dati: nella Cna, su 6.231 dipendenti totali, 4.622 si occupano di erogazione di servizi mentre i restanti 1.609 svolgono attività più propriamente sindacale; nella Confartigianato, su 6.401 dipendenti totali, $3.110 \mathrm{si}$ occupano di servizi e 3.291 di attività sindacale. In realtà le percentuali fornite dalla Cna sono assai più precise di quelle della Confartigianato, in quanto quest'ultima associazione è stata in grado di conteggiare i dipendenti adibiti ai servizi utilizzando il numero di dipendenti delle società di servizio promosse, per ragioni fiscali, dalle singole associazioni di primo grado aderenti alla confederazione. Un'ulteriore percentuale dei 3.291 dipendenti stimati per l'attività sindacale, pur se impiegata direttamente in associazione, si occupa tuttavia anch'essa di servizi. Al di là della maggiore o minore precisione assoluta delle cifre, il dato sostanziale è costituito dalla rilevanza organizzativa della funzione di erogazione dei servizi.

25 Per completare e chiarire il precedente parallelo con la Confindustria è forse utile sottolineare che quest'ultima eroga pochi o nulli servizi ai suoi associati, tant'è vero che gli organici delle sue articolazioni territoriali sono poca cosa rispetto a quelli delle altre associazioni imprenditoriali: il commerciante, l'agricoltore, l'artigiano che si associano vogliono essere rappresentati ma al tempo stesso hanno bisogno di essere supportati in tutte quelle funzioni che non presidiano internamente per ragioni di scala; al contrario chi si associa a Confindustria ricerca prevalentemente uno status sociale, tant'è vero che è disposto a pagare quote di tesseramento che, a parità di dimensioni e 
fatti ricordare che l'attività di erogazione di servizi si pone sul mercato, rispondendo ad una «razionalità» di carattere economico ed in ciò differendo dalla rappresentanza di interessi che risponde ad una razionalità di tipo politico (Zan 1992). Pertanto, se le associazioni, in quanto organizzazioni, nascono aderendo al modello del partito politico di massa (tramite sezioni, cellule, mandamenti per il presidio territoriale) e alla sua razionalità, nel tempo si trasformano anche in efficienti centri di servizio costituendo così uno strano ibrido organizzativo che non trova analogie in altre organizzazioni e costituisce davvero la peculiarità del modello di organizzazione associativa. Ma la presenza contemporanea di diverse razionalità, culture e strumenti gestionali produce un duplice impatto: da un lato l'attività economicamente fondata di erogazione di servizi ha generato una capacità di autoalimentazione e di generazione di risorse superiore a quella sindacale, ampliando via via la forbice sul piano dei criteri culturali, gestionali e strategici prevalenti nell'associazione e nel suo modello di sviluppo; dall'altro, nel rapporto con l'ambiente esterno, ha contribuito ad inasprire il rapporto di concorrenza con le altre associazioni di categoria, sostituendo agli steccati ideologici la competizione di mercato, e alla necessità della mediazione politica il confronto oggettivo sul rapporto qualità-prezzo come precondizione per la sopravvivenza. Tutto ciò è importante in quanto dimostra che non si può assumere la dimensione politico-ideologica come fattore esogeno monocausale in grado di spiegare la specificità dell'associazionismo imprenditoriale italiano, così come si è venuto evolvendo negli ultimi quarant'anni. Al contrario il fattore servizi appare una dimensione importante nello spiegare sia la storica mancanza di cooperazione intercategoriale, sia perché alla progressiva decadenza della polarizzazione ideologica sia subentrata un'attenuazione molto parziale del conflitto interorganizzativo ${ }^{26}$.

di settore dell'impresa, sono anche da 10 a 20 volte superiori. Ciò dimostra ulteriormente la differente e superiore capacità identificativa di Confindustria di cui abbiamo parlato sopra.

$26 \mathrm{E}$ evidente che tecnostrutture che si compongono di migliaia di persone e generano centinaia di miliardi di entrate costituiscono un vincolo formidabile ad ogni progetto politico che vada verso alleanze, unità politiche o unificazioni organiche; da questo punto di vista anche i sindacati confederali costituiscono un esempio rilevante e il continuo rinvio del progetto di unità sindacale trova spiegazioni nella diversa concezione non solo del modello sindacale ma anche nelle strategie di sviluppo delle attività di servizio del sindacato (l'esempio più recente è relativo all'assistenza previdenziale). 
Le tre dimensioni organizzative che ci siamo qui limitati a prendere in considerazione - gerarchia nominale, identità debole, fattore servizi - non esauriscono certo l'analisi delle associazioni imprenditoriali come attori organizzativi ed è ovvio che accanto ad esse sarebbe possibile citare molte altre variabili cui ciascuna associazione risponde in termini di specificità strutturale ed organizzativa o di idiosincrasia culturale: il rapporto tra articolazioni orizzontali e verticali, i meccanismi di elezione e legittimazione della dirigenza, il peso dei principi di espressione democratica diretta degli associati rispetto a quella delegata a politici di professione. Tuttavia, le risposte organizzative a questo tipo di problemi possono variare moltissimo tra le diverse associazioni ed anche all'interno di ciascuna, ed un'analisi di questo tipo non risponde ai fini di questo scritto. Al contrario quelle sopra esposte costituiscono a nostro parere tre dimensioni «forti», comuni a tutte le associazioni imprenditoriali qui considerate, soprattutto significative per descrivere i loro tratti salienti e per comprendere nell'attuale transizione quali siano i contesti che generano le scelte politiche o $\mathrm{i}$ vincoli che vi si oppongono.

Le trasformazioni dell'associazionismo imprenditoriale: alcune domande senza risposta

Nel corso della trattazione abbiamo individuato due caratteristiche particolarmente importanti delle associazioni imprenditoriali italiane: da un lato, la loro identità, e quindi la determinazione dei confini, è stata storicamente vincolata dal collateralismo partitico; dall'altro, la loro identità è debole perché fondata su confini largamente inclusivi dove le ragioni di membership faticano a trovare reciproca coerenza. Queste caratteristiche spiegano perché la crisi del collateralismo abbia procurato così grandi difficoltà alla maggior parte delle associazioni imprenditoriali e assai minori alla Confindustria (per molti anni quantomeno vicina alla Dc tramite i governi da essa espressi) o ai sindacati confederali (che possono permettersi il lusso di schierarsi in modo esplicito e di prendere al tempo stesso le distanze dal governo). Pertanto, anche qualora le associazioni imprenditoriali infrangessero quella sorta di tabù culturale che è l'orientamento collateralistico ed optassero per lo sviluppo di una cultura dell'autonomia, tale scelta non definirebbe in sé alcuna strategia politica. Debolezza significa infatti di per sé man- 
canza di autonomia e nessuna delle associazioni qui considerate può realisticamente ipotizzare di costruire la propria autonomia nel quadro frammentato, disgregato e spesso conflittuale dell'attuale sistema di rappresentanza imprenditoriale. In tal senso, il dibattito in corso in seno a molte associazioni, tra i gruppi dirigenti così come tra gli iscritti, è centrato sul problema dell'identità e dei confini storicamente tracciati tra una categoria economica e l'altra e tra le organizzazioni all'interno di ciascuna categoria. Il nucleo centrale della questione consiste dunque nella definizione delle coordinate che meglio consentono di individuare e integrare gli interessi ${ }^{27}$.

Le odierne associazioni imprenditoriali sono, in effetti, organizzazioni «vecchie», ancora troppo fortemente ancorate ai giochi strategici del passato in un'arena politica le cui regole sono invece strutturalmente mutate. I motivi di tale «obsolescenza» sono profondi e non banali, come si è messo in luce in questo lavoro. La ricerca di nuove coerenze sistemiche da parte associativa impone tuttavia un comportamento più «razionale», maggiormente aderente alla nuova struttura dei giochi. Poiché le possibilità di esercitare maggiore influenza dipendono dal conseguimento di una maggiore rappresentatività (effettiva, non nominale) la capacità delle varie espressioni dell'azione collettiva organizzata di generare e costruire integrazione sociale si presenta come la questione centrale. Alla stessa stregua dei partiti politici, le associazioni soffrono di una sorta di «deficit di ideologia».

Gli ultimi decenni della storia italiana ci hanno abituati ad una «società di mezzo» fortemente ideologizzata e ancillare rispetto al livello partitico-esecutivo. Tale condizione ha condizionato la costruzione delle élites, vincolandola al principio della «disciplina di partito» e limitandone un'autonoma evoluzione, elaborazione, innovazione. Il beneficio di una forte capacità di integrazione sociale è stato perciò pagato col limitato sviluppo di principi e valori etici, civici, deontologici relativi sia alle comuni regole della polis che a specifici segmenti o settori sociali e professionali. Alcuni importanti ordini professionali, e

27 Vale la pena sottolineare che il dibattito su questa questione centrale, vivo in alcune associazioni già da alcuni anni, ha subito un'evidente accelerazione dopo la formazione del governo Prodi. Mai, nella storia della Repubblica, una personalità di rilievo partitico o governativo si era rivolta con insistenza e in più occasioni all'insieme delle associazioni di categoria auspicando «la razionalizzazione complessiva del sistema». 
perfino il potere autonomo della magistratura, costituiscono oggi altrettanti e clamorosi esempi. La società di mezzo si è largamente sottratta ad un ruolo autonomo sul versante della pedagogia sociale ed in effetti non poteva non essere questo l'esito di una polarizzazione ideologica accentuata in seno alla società ed assai meno netta nel governo e nella regolazione degli interessi. Il declino delle ideologie e i prodromi della transizione ci hanno consegnato associazioni di rappresentanza deboli nell'elaborazione di valori e nella costruzione di integrazione sociale, eppure con una dote intatta, cioè una legittimazione sociale non incrinata ed anzi accentuata dalla domanda di rappresentanza generata dalla transizione (non è infatti per nulla paradossale che la «dis-integrazione» sociale accresca la richiesta di difesa dell'interesse più specifico, localistico o corporativo). Gli storici fattori di debolezza, tuttavia, si ripresentano come vincoli che rendono difficile alle associazioni giocare giochi nuovi e moltiplicano gli impacci e le inerzie derivanti dagli apparati professionali, dai gruppi dirigenti, dalle sub-culture storicamente antagoniste ed ancora reciprocamente diffidenti. In tutto ciò sta il peso attuale del fattore organizzativo.

La domanda di rappresentanza ha imposto, al tempo stesso, la ridefinizione delle identità collettive. Il fenomeno riguarda tutte le principali associazioni di rappresentanza e si sostanzia, nei due casi più noti agli osservatori, nella questione della difficile unità sindacale e nella scelta (rischiosa e, ad oggi, delegittimante) della rappresentanza generale dell'impresa da parte di Confindustria. Nelle altre associazioni imprenditoriali, i connotati del dibattito sono altrettanto ben definiti e vertono su una dimensione a tutto preliminare: la costruzione di nuovi valori. $\mathrm{Da}$ questo punto di vista, è interessante osservare che in molti settori (l'artigianato, la piccola impresa, l'agricoltura, la cooperazione) è in corso un dibattito incentrato proprio sulla ricerca di valori guida. A tale proposito, si fronteggiano due visioni diverse ed antagoniste, che tuttavia non contrappongono tra loro associazioni «ex-rosse» ed «ex-bianche», ma costituiscono piuttosto terreno di confronto anche interno a ciascuna singola associazione. Una visione fa perno su un approccio fondamentalmente economico della rappresentanza d'impresa, l'altra su un approccio fondamentalmente sociale.

Coloro che aderiscono alla teoria economica fanno proprio il classico principio utilitarista, e pongono a proprio riferimento, e come soggetto rappresentato, l'impresa in senso pieno. Lo 
scenario in questo caso è il mercato, e la rappresentanza verte sulla regolazione del mercato. Tale visione è condivisa da organizzazioni diverse tra loro (ad esempio la Cna, la Confapi, la Lega delle Cooperative) ma con un obiettivo politico simile: rappresentare l'impresa di mercato, attiva, evoluta (sia essa piccola impresa, piccola industria, impresa cooperativa) e configurarsi in tal modo come soggetto politico contrapposto a quello confindustriale (identificato come rappresentante della grande impresa tradizionale, privata e pubblica) e a quello sindacale del lavoro dipendente.

Le associazioni che aderiscono prevalentemente alla teoria sociale scelgono come soggetto di riferimento e di rappresentanza non l'impresa ma l'individuo imprenditore. Lo scenario è in questo secondo caso la società stessa e la rappresentanza verte sulla regolazione sociale e sulla collocazione sociale dell'imprenditore. Anche tale visione accomuna organizzazioni tra loro diverse (ad esempio la Confcommercio, la Coldiretti, la Confcooperative) che condividono l'obiettivo di proporsi come rappresentanze dei ceti medi produttivi e del lavoro autonomo, contrapposte all'interdipendenza reciproca del capitale e del lavoro dipendente.

La prima concezione ha come riferimento l'organizzazione e la tutela degli interessi dell'impresa e delle leggi di mercato ed è interessante che vi si riconoscano tanto organizzazioni che mirano a rappresentare le imprese di piccole dimensioni (dove è forte la compenetrazione tra il lavoro dell'imprenditore ed i fattori di rischio mediante la sua partecipazione diretta), quanto organizzazioni che rappresentano da sempre le istanze più grandi ed evolute imprese cooperative (in primis quelle di consumo nella grande distribuzione). La seconda concezione ha come riferimento il lavoro autonomo - concepito innanzi tutto come soggetto sociale da tutelare, nelle sue molteplici versioni e come vettore di conoscenze, capitale o lavoro diretto. Anche in questo caso è interessante che in tale visione si riconoscano associazioni assimilabili dalla difesa di soggetti imprenditoriali che, per motivi differenti di intrinseca debolezza, non si riconoscono nella pura logica di mercato: ad esempio la piccola distribuzione, una parte delle imprese agricole, le piccole imprese cooperative.

Se teniamo presente questo fondamentale crinale ideologico possiamo ancor meglio comprendere perché, fino ad oggi, l'«implausibilità del modello neo-collaterale» non sia stata in 
grado di produrre cambiamenti organizzativi rilevanti nel sistema della rappresentanza funzionale. Le diversità su questi valori di fondo hanno impedito la scelta in apparenza più ovvia: l'unità politica di categorie omogenee. Possiamo inoltre spiegare meglio le ambiguità, le contraddizioni e le lentezze che tali processi, comunque in atto, stanno dimostrando, partendo dai contesti locali per arrivare a quelli nazionali. Se il problema centrale delle associazioni continua ad essere il ristabilire nuove coerenze sistemiche, possiamo ipotizzare che oggi le varie associazioni siano tese alla definizione di nuovi «valori non negoziabili» (Pizzorno 1983), non più demandati al sistema partitico, ma piuttosto autonomamente elaborati. D'altronde, sono proprio i valori non negoziabili gli elementi di maggiore rigidità all'interno dell'arena politica. Di conseguenza, le due visioni sopra presentate assumono un duplice significato: da un lato, costituiscono in sé un elemento di analisi degli assetti attuali e concorrono a spiegare alcune dinamiche; dall'altro, spingono ad interrogarsi sul loro possibile impatto rispetto all'evoluzione in corso.

In linea di principio, possiamo notare due aspetti. Da un lato, la complementarità dei due approcci, quello economico e quello sociale; dall'altro, il fatto che le attuali regole del gioco nella regolazione degli interessi avvantaggiano le categorie produttive altamente rappresentative su scala nazionale e con un forte presidio organizzativo centrale. Questi due fattori potrebbero rinforzarsi a vicenda nel dettare le regole di un'evoluzione possibile più «razionale»?

Non è possibile fornire risposte a domande di questo tipo, considerando l'aleatorietà e l'incertezza del nostro sistema istituzionale. Se il prossimo futuro dovesse riservare un mutamento in senso federalista, ciò renderebbe i ragionamenti condotti assai meno rilevanti, non foss'altro perché acquisirebbe plausibilità l'ipotesi di un'ulteriore frammentazione della rappresentanza, in antitesi a grandi centrali confederali, ed in senso policentrico. La scala locale (provinciale o regionale) potrebbe cioè diventare luogo privilegiato della regolazione e dell'integrazione socio-economica ${ }^{28}$. A conclusioni opposte si potrebbe invece giungere esaminando la natura ed il contenuto delle politiche che caratterizzano e, presumibilmente, per i prossimi anni caratterizzeranno l'azione del decisore pubblico: il fatto, cioè, che

28 Accade sovente che articolazioni territoriali particolarmente forti e rilevanti tendano a ricercare rapporti diretti con i ministeri scavalcando il loro livello nazionale. 
siano centrate assai più sull'allocazione di costi che di risorse alle varie parti sociali. E noto che le scelte relative alle entrate statali discriminano soprattutto per settori economici di attività e categorie sociali e solo in casi particolari, ed in maniera minimale, per aree geografiche. Com'è ben evidente, l'influenza dei gruppi di interesse è soprattutto orientata a minimizzare la rispettiva partecipazione ai costi del risanamento del debito pubblico. Pertanto, l'arena politica ha oggi una scansione assai più verticale che orizzontale ed è questo l'asse che gli attori in gioco tendono a privilegiare.

Le due visioni, alle quali ci siamo riferiti come approccio economico e approccio sociale, sono utili soprattutto per comprendere la rilevanza della discriminante ideologica come fattore di aggregazione organizzativa, o comunque di ordine coalizionale, in relazione alla struttura dei giochi nell'arena politica attuale. Le due visioni appaiono più complementari che reciprocamente escludentisi ed in questo senso sembrano costituire una sorta di assi primari a partire dai quali scaturiscono il confronto, lo scontro e la ricerca di egemonia da parte dei vari attori nella ridefinizione delle identità funzionali. La prima visione, l'approccio economico, se implica una maggiore tensione corporativa all'interno di una concezione tradizionale della rappresentanza imprenditoriale, seleziona e ricomprende le istanze del tessuto imprenditivo evoluto (della piccola industria, dell'artigianato, dell'agricoltura, del terziario) che abbisogna di una rappresentanza organica e sistemica delle interrelazioni orizzontali e verticali su cui si fonda, in contrapposizione e competizione direttamente con la grande industria ed al di fuori di ogni rapporto di subalternità così come si è storicamente determinato in Italia in molti settori. La seconda visione, l'approccio sociale, fondandosi sulla rappresentanza dei ceti medi produttivi e del lavoro autonomo, presuppone un allargamento della sfera degli interessi rappresentati in un'ottica più generale, quasi a somiglianza del modello sindacale dopo la svolta dell'Eur del 1978. Si tratta, in effetti, di una sorta di sindacalismo del lavoro autonomo, in quanto esteso all'intera pluralità dei soggetti potenzialmente tutelabili, e quindi anche ai più deboli. Il problema di fondo è che oggi, in quasi tutte le associazioni imprenditoriali, le due visioni convivono e, con esse, le relative contraddittorie istanze. Ciò rende difficoltoso non solo il processo di ridefinizione degli assetti complessivi, ma anche lo stesso processo di negoziazione e definizione delle principali politiche pubbliche da parte dell'esecutivo. 
L'incertezza del contesto istituzionale; le contraddizioni e le complessità insite nel sistema della rappresentanza imprenditoriale; il venir meno, nell'arena politica, di regole del gioco profondamente radicate; la difficoltà di farne emergere di nuove: sono questi i principali elementi del quadro attuale dell'associazionismo imprenditoriale le cui possibilità di evoluzione costituiscono per ora domande senza risposta.

\section{Riferimenti bibliografici}

Addario, N. e P. Segatti (1982), L'organizzazione dell'azione collettiva degli imprenditori, in «Rassegna Italiana di Sociologia», n. 1.

Amato, G. (1976), Economia, politica ed istituzioni, Bologna, Il Mulino.

Bordogna, L. e G. Provasi (1984), Politica, economia e rappresentanza degli interessi, Bologna, Il Mulino.

Cartocci, R. (1990), Elettori in Italia, Bologna, Il Mulino.

- (1994), Fra Lega e Chiesa, Bologna, Il Mulino.

Cnel (1996), Laboratori territoriali, competizione e leadership nella questione settentrionale, Roma.

Cotta, M. e P. Isernia (a cura di) (1996), Il gigante dai piedi d'argilla, Bologna, Il Mulino.

Crozier, M. e H. Friedberg (1977), L'acteur e le systéme, Paris, Seuil, trad. it. Attore sociale e sistema, Milano, Etas Libri, 1978.

Diamanti, I. (1996a), Per la Lega tre anime ma la stessa sfida, in «Il Sole 24 Ore», 23 aprile.

- (1996b), Ma Bossi ba uno zoccolo duro, in «L'Espresso», 26 settembre.

Diamanti, I. e R. Mannheimer (a cura di) (1994), Milano a Roma. Guida all'Italia elettorale del 1994, Roma, Donzelli.

Elster, J. (1979), Ulysses and the Sirens, Cambridge, Cambridge University Press, trad. it. Ulisse e le sirene, Bologna, Il Mulino, 1983.

Feltrin, P. (1994), Unità, autonomia, impegno politico: tre facce di una stessa moneta, in «Prospettiva Sindacale», n. 86.

Feltrin, P. e S. Zan (1995), La transizione di regime in Italia, Paper dell'Osservatorio sulle Associazioni di Rappresentanza, Università di Bologna.

Ferrante, M. (1993), Il consumatore imprenditore, Bologna, Il Mulino.

Friedberg, H. (1993), Le pouvoir et la regle. Dynamiques de l'action organisée, Paris, Seuil, trad. it. Il potere e la regola. Dinamiche dell'azione organizzata, Milano, Etas Libri, 1994.

Hirschman, A. (1970), Exit, Voice and Loyalty, Cambridge, Harvard University Press, trad. it. Lealtà, defezione, protesta, Milano, Bompiani, 1982. 
Katz, R.S. (1987), Party Government: European and American Experiences, Berlin, New York, De Gruyter.

Lange, P. e M. Regini (a cura di) (1987), Stato e regolazione sociale, Bologna, Il Mulino.

Lanzalaco, L. (1990), Dall'impresa all'associazione. Le organizzazioni degli imprenditori: la Confindustria in prospettiva comparata, Milano, Angeli.

Lassini, A. (1984), Rappresentanza, autonomia organizzativa e legittimazionismo imprenditoriale, in «Stato e Mercato», n. 11.

Lawrence, P.R. e J.W. Lorsch (1967), Organization and Environment, Cambridge, Harvard University Press.

Lijphart, A. (1969), Consociational Democracy, in «World Politics», XXI.

Mannheimer, R. e G. Sani (1987), Il mercato elettorale, Bologna, Il Mulino.

Martinelli, A. (a cura di) (1994), L'azione collettiva degli imprenditori italiani, Roma, Comunità.

Mattina, L. (1991), Gli industriali e la democrazia. La Confindustria nella formazione dell'Italia repubblicana, Bologna, Il Mulino.

- (1994a), Gli attori economici. Vincitori e vinti, in Diamanti e Mannheimer (a cura di).

- (1994b), I candidati, in «Rivista Italiana di Scienza Politica», XXIV, n. 3.

Morlino, L. (a cura di) (1991), Costruire la democrazia, Bologna, Il Mulino.

O' Connor, J. (1973), The Fiscal Crisis of the State, New York, St. Martin's Press, trad. it. La crisi fiscale dello stato, Torino, Einaudi, 1977.

Olson, M. (1965), The Logic of Collective Action, Cambridge, Harvard University Press, trad. it. La logica dell'azione collettiva, Milano, Feltrinelli, 1983.

Ouchi, W. (1980), A Framework for Understanding Organizational Failure, in J.R. Kimberly e R.H. Miles (a cura di), The Organizational Life-cycle, San Francisco, Jossey-Bass.

Panebianco, A. (1982), Modelli di partito, Bologna, Il Mulino.

Pasquino, G. (1987), Regolatori sregolati, in Lange e Regini (a cura di).

Pizzorno, A. (1983), Il sistema pluralistico di rappresentanza, in S. Berger (a cura di), L'organizzazione degli interessi nell'Europa occidentale, Bologna, Il Mulino.

- (1993), Le radici della politica assoluta, Milano, Feltrinelli.

Regini, M. (1981), I dilemmi del sindacato, Bologna, Il Mulino.

Salvadori, M. (1996), Stati e democrazia nell'era della globalizzazione, in «il Mulino», n. 3.

Sani, G. (1992), La destrutturazione del mercato elettorale, in «Rivista Italiana di Scienza Politica», XXII, n. 2. 
Sartori, G. (1966), European Political Parties: the Case of Polarized Pluralism, in J. La Palombara e M. Wiener (a cura di), Political Parties and Political Development, Princeton, Princeton University Press.

- (1982), Teoria dei partiti e caso italiano, Milano, Sugarco.

Selznick, P. (1957), Leadership in Administration, New York, Harper \& Row, trad. it. La leadership nelle organizzazioni, Milano, Angeli, 1976.

Speroni, D. (1975), Il romanzo della Confindustria. Dal 1910 al 1975, Milano, Sugarco.

Urbani, G. (1977), Atteggiamenti politici degli imprenditori: il caso della Lombardia, in «Rivista Italiana di Scienza Politica», VII, n. 1.

Williamson, O. (1975), Markets and Hierarchies: Analysis and Antitrust Implications, New York, The Free Press.

Windmuller, J.P. e A. Gladstone (a cura di) (1985), Le organizzazioni degli imprenditori, Roma, Ed. Lavoro.

Zan, S. (1982), La cooperazione in Italia, Bari, De Donato.

- (1992), Organizzazione e rappresentanza, Roma, La Nuova Italia Scientifica. 